Article

\title{
Assessment of the Effects of Bisphenol A on Dopamine Synthesis and Blood Vessels in the Goldfish Brain
}

\author{
Qing Wang ${ }^{1,2}$, Fangmei Lin ${ }^{1}$, Qi He ${ }^{1}$, Xiaochun Liu ${ }^{1}$, Shiqiang Xiao ${ }^{1}$, Leyun Zheng ${ }^{3}$, \\ Huirong Yang ${ }^{1,2, *}$ and Huihong Zhao ${ }^{1,2, *}$ \\ 1 College of Marine Sciences, South China Agricultural University, Guangzhou 510642, China; \\ wangqing@scau.edu.cn (Q.W.); linmu212422@163.com (F.L.); 13657405840@163.com (Q.H.); \\ zhuifeng2019yun@163.com (X.L.); xiaosq01@haid.com.cn (S.X.) \\ 2 Joint Laboratory of Guangdong Province and Hong Kong Region on Marine Bioresource Conservation and \\ Exploitation, College of Marine Sciences, South China Agricultural University, Guangzhou 510642, China \\ 3 Fisheries Research Institute of Fujian, Xiamen 361000, China; heicaonan@163.com \\ * Correspondence: hry@scau.edu.cn (H.Y.); zhaohh@scau.edu.cn (H.Z.)
}

Received: 18 September 2019; Accepted: 4 December 2019; Published: 9 December 2019

\begin{abstract}
Bisphenol A (BPA) is an abundant contaminant found in aquatic environments. While a large number of toxicological studies have investigated the effects of BPA, the potential effects of BPA exposure on fish brain have rarely been studied. To understand how BPA impacts goldfish brains, we performed a transcriptome analysis of goldfish brains that had been exposed to $50 \mu \mathrm{g} \mathrm{L}-1$ and $0 \mu \mathrm{g}$ $\mathrm{L}^{-1} \mathrm{BPA}$ for 30 days. In the analysis of unigene expression profiles, 327 unigenes were found to be upregulated and 153 unigenes were found to be downregulated in the BPA exposure group compared to the control group. Dopaminergic signaling pathway-related genes were significantly downregulated in the BPA exposure group. Furthermore, we found that serum dopamine concentrations decreased and TUNEL (terminal deoxynucleotidyl transferase 2-deoxyuridine, 5-triphosphate nick end labeling) staining was present in dopamine neurons enriched regions in the brain after BPA exposure, suggesting that BPA may disrupt dopaminergic processes. A KEGG analysis revealed that genes involved in the fluid shear stress and atherosclerosis pathway were highly significantly enriched. In addition, the qRT-PCR results for fluid shear stress and atherosclerosis pathway-related genes and the vascular histology of the brain showed that BPA exposure could damage blood vessels and induce brain atherosclerosis. The results of this work provide insights into the biological effects of BPA on dopamine synthesis and blood vessels in goldfish brain and could lay a foundation for future BPA neurotoxicity studies.
\end{abstract}

Keywords: bisphenol A; transcriptome; dopamine; signaling pathway; brain blood vessels

\section{Introduction}

Bisphenol A (BPA) is an environmental contaminant that is widely used in many consumer goods. For example, it is used in coatings on food cans, thermal receipt paper, and water bottles [1,2]. It is estimated that the production of the biosurfactant market could increase to 460 million kilograms by 2020 , indicating that approximately $450,000 \mathrm{~kg}$ of BPA will be released into the environment [3]. BPA levels are very high in water worldwide. For example, levels of BPA in surface water in the Netherlands reached $21 \mu \mathrm{g} \mathrm{L}^{-1}$ in the summer [4]. The level in landfill leachate from Japan was as high as $17.2 \mathrm{mg} \mathrm{L}^{-1}$ [5]. Owing to its release from used polycarbonate animal cages, the concentration of BPA increased to $310 \mu \mathrm{g} \mathrm{L}^{-1}$ [6]. In China, BPA levels in water are also up to $370 \mu \mathrm{g} \mathrm{L}^{-1}[7,8]$. The aquatic environment contains large amounts of BPA, and this chemical can enter aquaculture 
water, mainly through the natural degradation of BPA-containing products and landfill leachate and sewage treatment wastewater contamination [9-14].

Fish are often used to evaluate the health of aquatic ecosystems, in particular, physiological and biochemical changes in fish are considered biomarkers of environmental pollution [15]. Previous studies have suggested that BPA, as an analog of estrogen, may exert an estrogenic effect on fish gonad development and reproductive axis-related gene expression [16-20]. BPA also affects various biological processes in fish, including metabolism, individual cortisol stress responses, energy metabolism, muscle structure, motor behavior, and axonal growth, thereby affecting fish growth and development [21-24]. In mammals, studies have shown that BPA is neurotoxic and induces brain, region-specific changes in dopaminergic processes, resulting in hyperactivity and attention deficits $[25,26]$. While numerous toxicological studies have described the effects of BPA on fish, few have investigated the effects of BPA on fish brain.

Our previous study showed that $50 \mu \mathrm{g} \mathrm{L}{ }^{-1}$ BPA exposures exert an obvious physiological effect on goldfish, such as disrupted testis maturation and reduced ovarian maturation, by affecting the HPG (Hypothalamic-pituitary-gonadal) axis [19]. This concentration can also be detected in aquatic environments $[7,8]$. Thus, in the present study, we perform a deep transcriptome analysis of the brains of goldfish exposed to $50 \mu \mathrm{g} \mathrm{L}^{-1}$ BPA to investigate the effects of BPA on fish brain. The data reveal candidate pathways for genes associated with dopamine synthesis, brain blood shear stress, and atherosclerosis, and provide extensive information about novel gene sequences and gene expression profiles. Additionally, we analyze serum dopamine concentrations and the expression profiles of the dopaminergic signaling pathway-related genes tyrosine hydroxylase $(t h)$, monoamine oxidase (mao), dopamine 1 receptor ( $d r 1)$, dopamine 2 receptor $(d r 2)$, and dopamine transporter (dat). We also examine apoptosis in dopaminergic neurons with a terminal deoxynucleotidyl transferase 2 -deoxyuridine $5^{\prime}$-triphosphate nick end-labeling (TUNEL) assay. The results show that BPA can disrupt dopamine synthesis. Additionally, the expression profiles of blood shear stress and atherosclerosis genes, and the results of brain vascular histologic analysis, show that BPA may damage brain blood vessels. The results of this study will help guide further investigation into the dopamine system and brain blood vessels in goldfish.

\section{Results}

\subsection{Sequencing and Assembly}

All goldfish in the treatment group were treated with BPA for 30 days prior to any testing. Six cDNA libraries were constructed: Three control group and three treatment group libraries. A total of $126,926,073$ raw reads were generated. There were $62,210,962$ and $63,902,684$ clean reads in the control and treatment group libraries, respectively. The Q20 values were greater than $98 \%$, and the GC (GC base) content was 49\% (Table S1). The low-quality reads were removed, and the clean reads were used for sequence assembly. After processing with Trinity software, 533,674 unigenes were ultimately generated (Table S2). These results suggested that high-quality transcriptome data were obtained. The sequence data for the unigenes were deposited in the Sequence Read Archive (SRA) of NCBI under accession numbers SRR8439278 to SRR8439283.

\subsection{Functional Analysis}

To evaluate the functions of the unigenes, 380,155 unigene sequences were aligned with sequences in seven public databases. The number of successfully annotated unigenes was 25,738 for the $\mathrm{Nr}$ database, 74,201 for the KEGG database, 96,930 for the Swiss-Prot database, and 13,933 for the GO database (Table S3). 


\subsection{Analysis of Differential Gene Expression}

DESeq2 software was used to analyze the unigene data and to identify the genes with significant expression differences between the control group and the treatment group. Genes with expression fold changes $>2(p<0.05)$ were considered to be differentially expressed. A total of 481 genes were differentially expressed, including 327 upregulated genes and 153 downregulated genes (Figure 1). Among these DEGs (differentially expressed genes), the expressions of three dopaminergic signaling pathway-related genes, th, mao, and $d r 1$, were significantly lower in the BPA-exposed group than in the control group (Figure 3A). The details of the changes in DEGs between the groups are listed in Table S4.

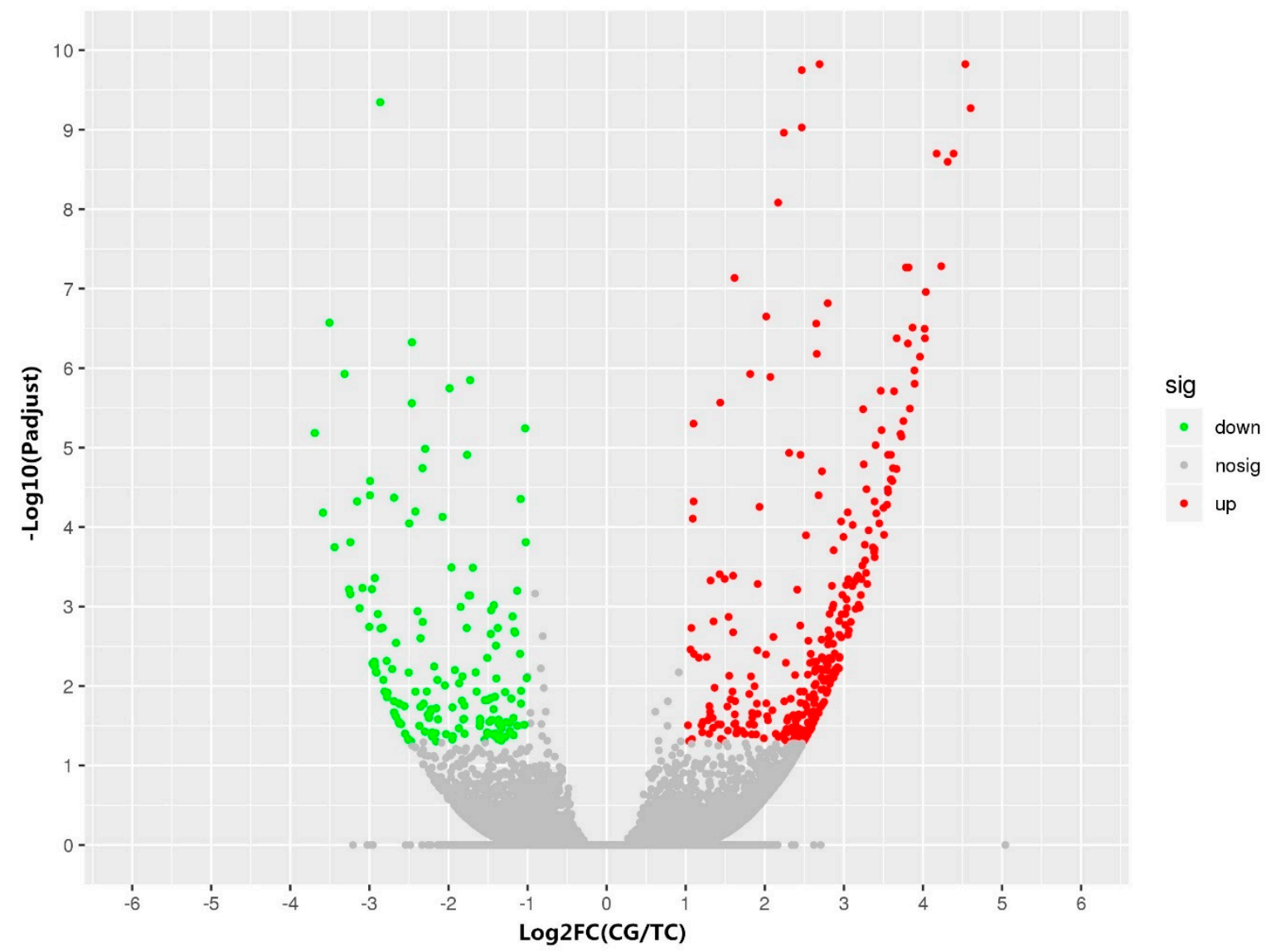

Figure 1. Volcano plot of the DEGs (differentially expressed genes) between the control group (CG) and the Bisphenol A (BPA) treatment group (TG). Log2FC (CG/TG) indicates the mean expression level ( $\log 2$ fold change) for each gene. Each dot represents a gene. The red dots represent upregulated genes, green dots represent downregulated genes, and gray dots represent genes that were not differentially expressed.

\subsection{GO and KEGG Enrichment Analysis}

To further analyze the DEGs between the control group and the BPA treatment group, the DEGs were annotated with the GO and KEGG databases. A total of 308 DEGs between the two groups were annotated with one or more GO terms (Figure S1). In the GO enrichment analysis, the most represented term in the biological processes category was the cellular process (GO: 0009987). In the cellular component category, the most strongly represented term was the extracellular region part (GO:0044421) term. In the molecular function category, nucleic acid binding transcription factor activity (GO:0001071) was the major term. A number of unigenes were also involved in metabolic processes, developmental processes, rhythmic processes, cell junctions, and catalytic activity, suggesting that these unigenes may play roles in mediating the effects associated with BPA exposure. 
In KEGG analyses, for any pairwise comparisons, the most highly enriched KEGG pathways were associated with circadian and immune processes; these pathways included the circadian rhythm, antigen processing and presentation, and herpes simplex infection pathways. In addition to these pathways, the fluid shear stress and atherosclerosis pathway was also highly significantly enriched (Figure 2).

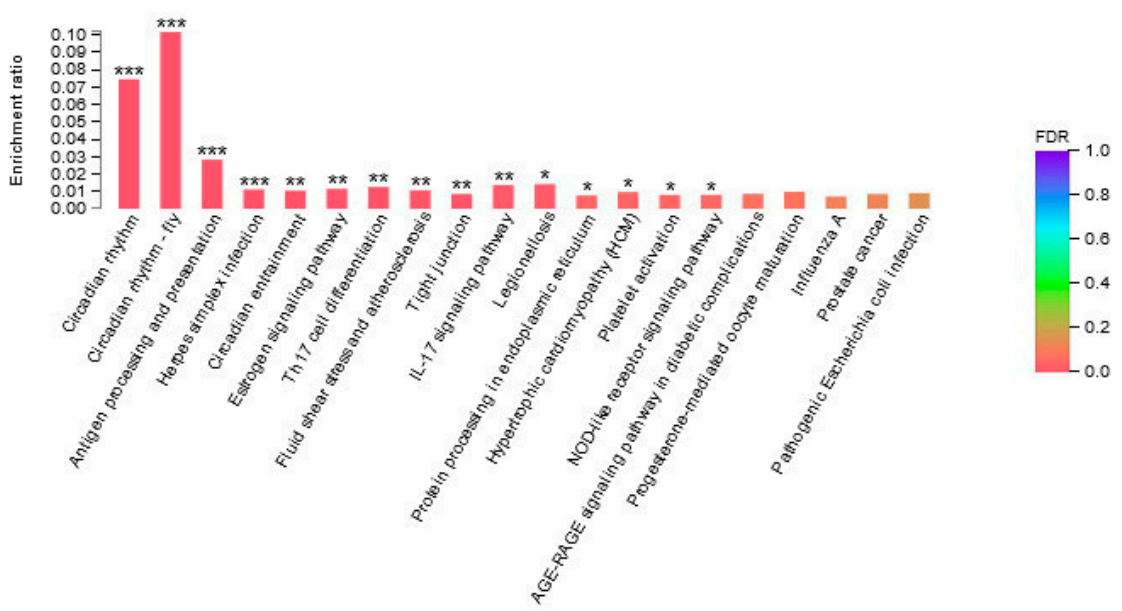

Figure 2. Enriched KEGG terms for the DEGs between the control group (CG) and the BPA treatment group (TG). * indicates a significant difference at $p<0.05,{ }^{* *}$ indicates a significant difference at $p<0.01$, and ${ }^{* * *}$ indicates a significant difference at $p<0.001$.

Among the DEGs in the fluid shear stress and atherosclerosis pathway, hsp90 and calm, which are associated with endothelial cell (EC) caveolae and vascular smooth muscle contraction, were downregulated in the BPA exposure group. The $\beta$-catenin and mek 5 genes, which are associated with antiatherogenic processes, were also downregulated, as was F-actin, which is associated with cytoskeletal alignment. Et-1, which is associated with proatherogenic processes, was upregulated in the BPA exposure group (Figure 4A, Table S5, Figure S2).

\subsection{Expression Profiles of Genes in Dopaminergic Signaling Pathway and the Fluid Shear Stress and Atherosclerosis Pathway}

As described in the Introduction Section, the effects of BPA on dopaminergic pathways in mammals have been described in previous studies $[25,26]$. In the current study, we therefore focused on the BPA effects on dopaminergic pathways in fish. The expression levels of key genes in the dopaminergic signaling pathway and the fluid shear stress and atherosclerosis pathway, including $t h, d r 1, d r 2$, dat, mao, $\beta$-catenin, $h s p 90$, calm, mek5, F-actin, and et-1, were analyzed. The results showed that the expression of all of the dopaminergic signaling pathway-related genes, including th, $d r 1, d r 2$, dat, and mao, was significantly lower in the BPA exposure group than in the control group (Figure 3B). The fluid shear stress and atherosclerosis pathway-related genes, such as $\beta$-catenin, hsp90, calm, and F-actin, were significantly downregulated in the BPA exposure group compared to the control group, but the expression of mek5 and et-1 was increased after BPA exposure (Figure 4B). 


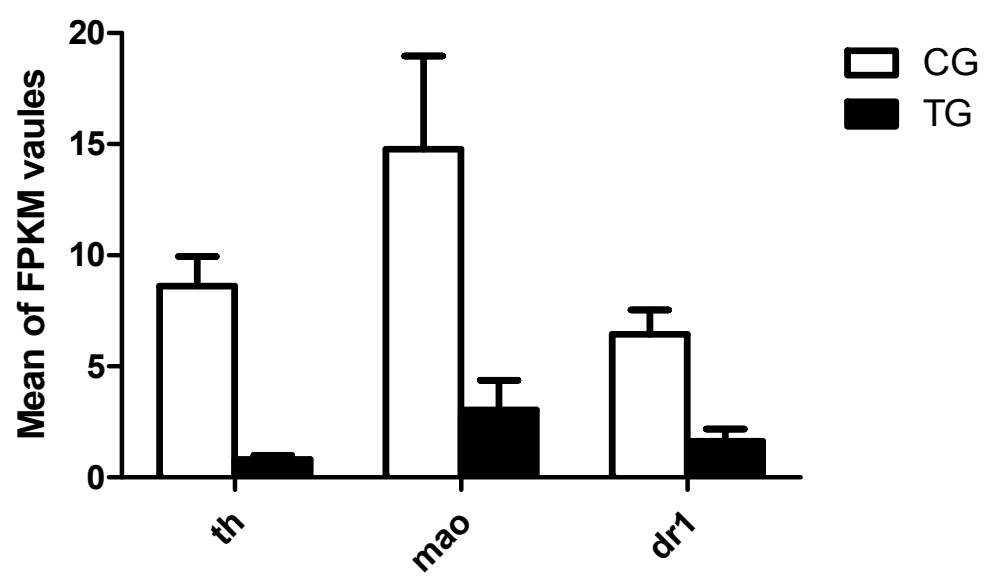

(A)

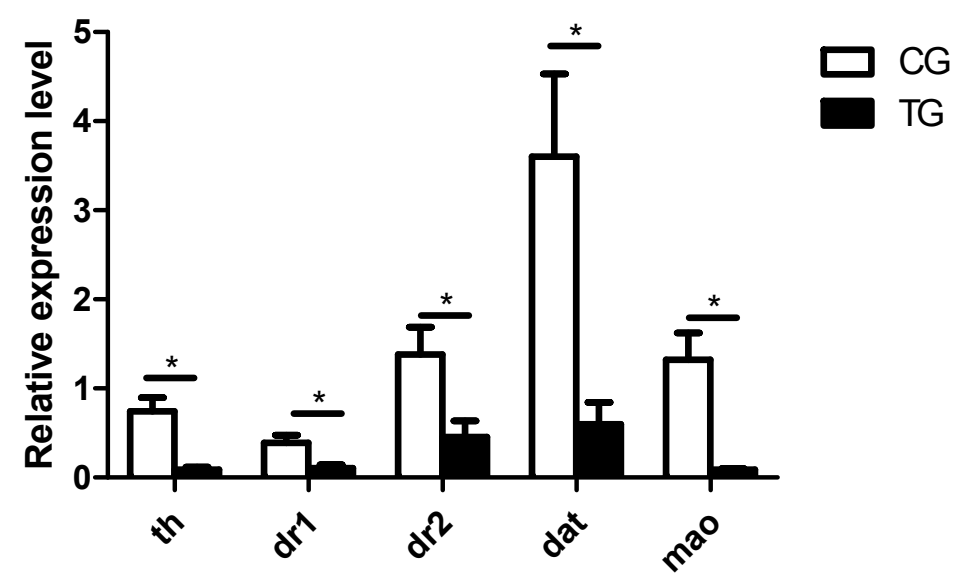

(B)

Figure 3. DEGs in the dopaminergic signaling pathway and analysis of the expression of dopaminergic signaling pathway-related genes by qRT-PCR. (A) Expression levels of 3 DEGs in the dopaminergic signaling pathway from the transcriptome data. (B) Expression levels of 6 dopaminergic signaling pathway-related genes determined by qRT-PCR $(n=3)$. CG: Control group; TG: BPA treatment group; and FPKM: Fragments per kilobase of transcript per million mapped reads. The qRT-PCR results were calculated from at least three independent experiments. EF1 was used as an internal normalization control. The data are expressed as the mean $\pm \mathrm{SD}\left({ }^{*} p<0.05\right)$.

\subsection{Analysis of Dopamine Concentrations and Brain Dopaminergic Neuron Apoptosis}

BPA has previously been shown to affect dopaminergic pathways in mammals $[25,26]$, and we showed here that DEGs involved in dopaminergic pathways were deregulated in goldfish after BPA exposure. To determine whether BPA affected dopaminergic production and dopaminergic neurons in goldfish in vivo, we examined the effects of BPA exposure on serum dopamine concentrations and stained brain sections with TUNEL. Serum dopamine concentrations were examined. Compared with those in the control group, dopamine concentrations were significantly decreased in the BPA-exposed group (Figure 5).

In the treatment group, positive TUNEL staining was found in the diencephalon area, the main site of dopaminergic neuron distribution (Figure 6D,F,H), and the number of TUNEL-positive cells per unit area was also higher in the BPA treatment group (Figure 6I-K), but no positive signals were observed in the corresponding area in the control group (Figure 6C,E,G). The main dopaminergic neuron distribution area has been described by Matsui [26] and Schweitzer [27]. The dorsal view of the fish brain is shown in Figure 6A, and the longitudinal sectional view is shown in Figure 6B. 


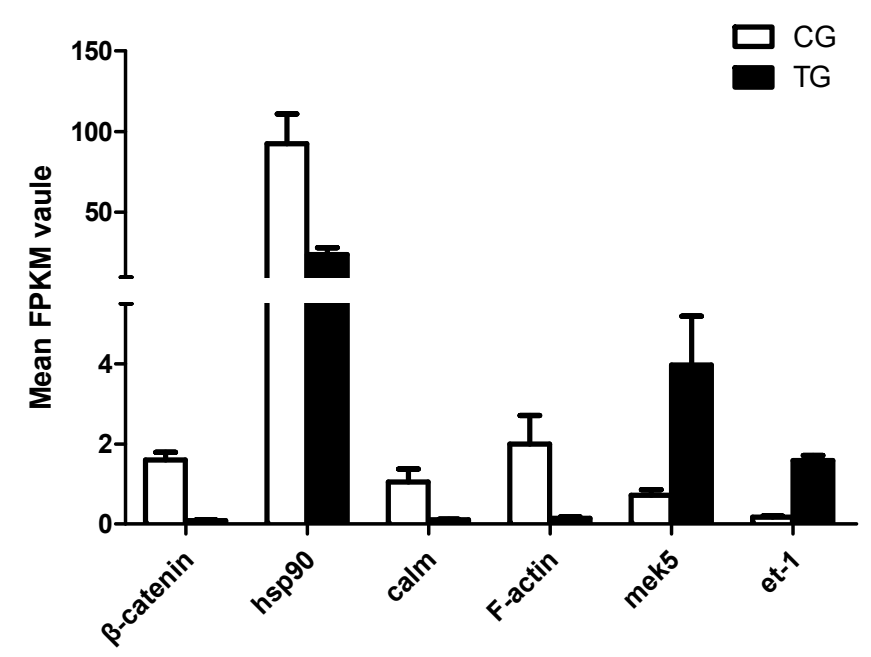

(A)

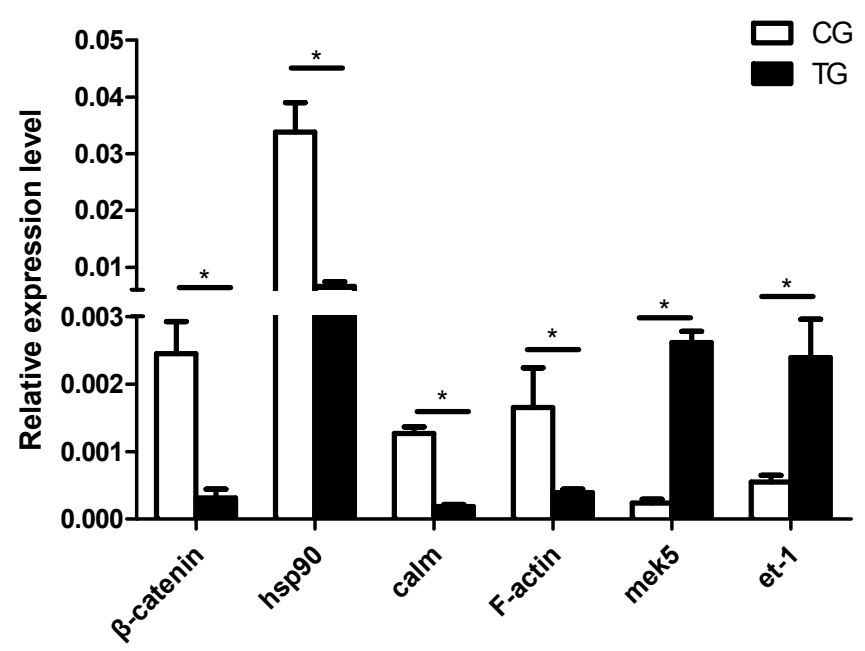

(B)

Figure 4. DEGs in the fluid shear stress and atherosclerosis pathway and validation by qRT-PCR. (A) Expression levels of 6 DEGs in the fluid shear stress and atherosclerosis pathway from the transcriptome data. (B) Validation of DEGs in the fluid shear stress and atherosclerosis pathway by qRT-PCR $(n=3)$. CG: Control group; TG: BPA treatment group; and FPKM: Fragments per kilobase of transcript per million mapped reads. The qRT-PCR results were calculated from at least three independent experiments. EF1 was used as an internal normalization control. The data are expressed as the mean $\pm \mathrm{SD}\left({ }^{*} p<0.05\right)$.

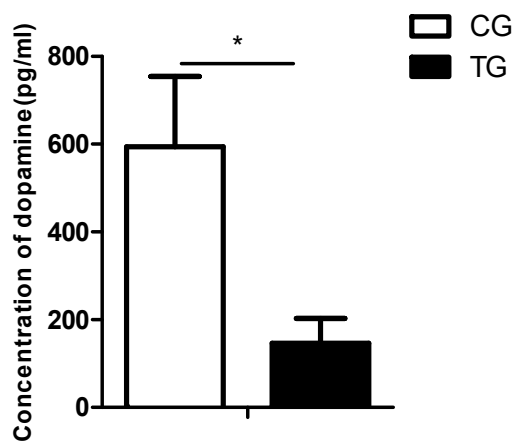

Figure 5. Serum dopamine concentrations in the control group (CG) and the BPA treatment group (TG). The data are expressed as the mean $\pm \mathrm{SD}(n=3) ;{ }^{*}$ indicates a significant difference at $p<0.05$. 

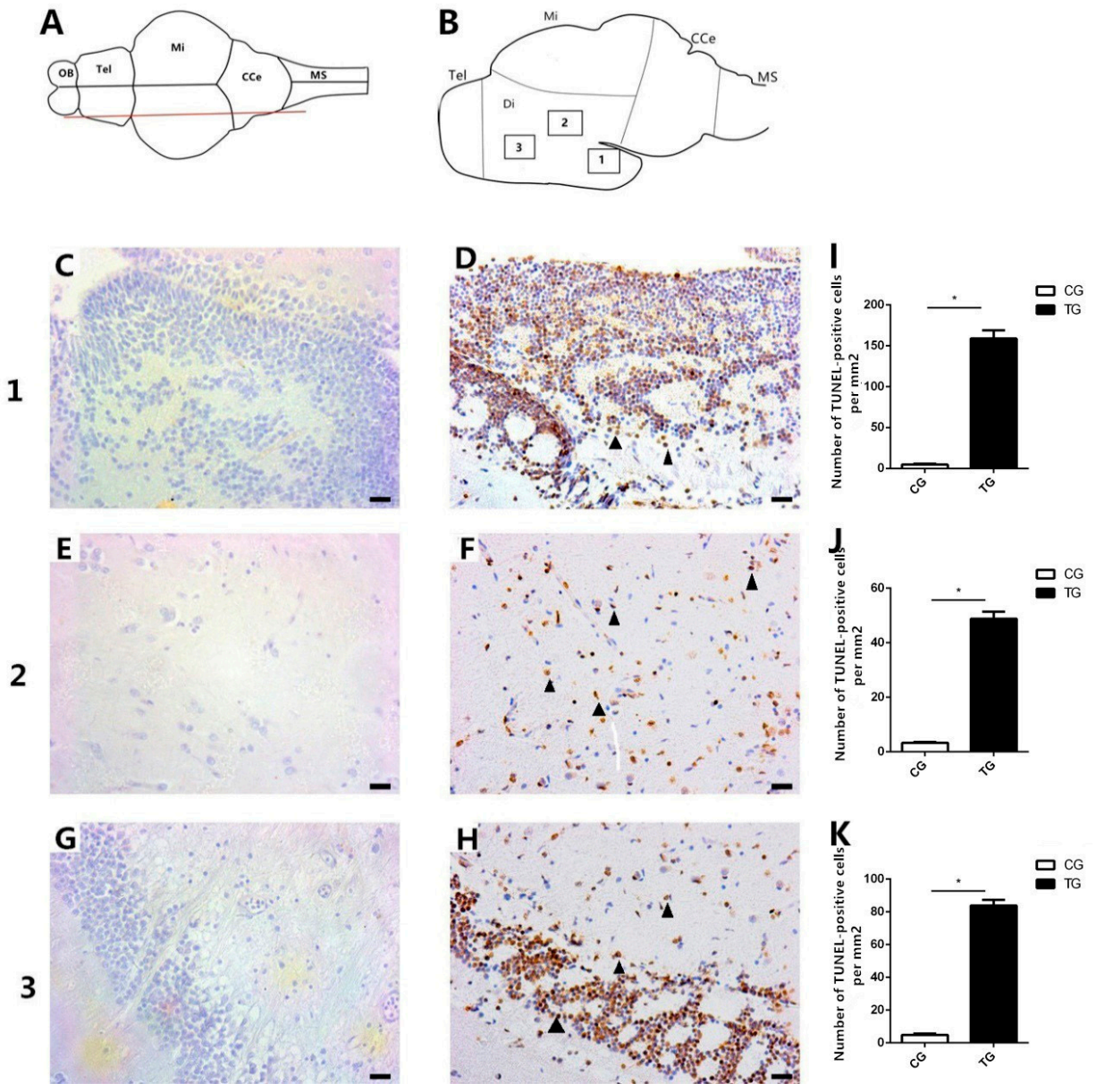

Figure 6. TUNEL staining of the brains of goldfish in the control group $\left(0 \mu \mathrm{g} \mathrm{L}^{-1}\right)$ and the BPA treatment group $\left(50 \mu \mathrm{g} \mathrm{L}^{-1}\right)$. (A) Dorsal view of the fish brain; the red line represents the slice position. (B) Longitudinal sectional view of the fish brain; the boxed areas represent the positions of the sections. (C-H) TUNEL staining of brain Section 1, Section 2, and Section 3. No TUNEL-positive signals were detected in the control group in the three positions $(\mathbf{C}, \mathbf{E}, \mathbf{G}) .(\mathbf{D}, \mathbf{F}, \mathbf{H})$ TUNEL-positive signals were detected in all three positions in the BPA-exposed group (black arrowheads). (I-K) Statistical analysis of the number of TUNEL-positive cells per $\mathrm{mm}^{2}$. Five images per fish were analyzed. * indicates a significant difference $(n=3)$. Tel: Telencephalon; OB: Olfactory bulb; Di: Diencephalon; Mi: Midbrain; CCe: Corpus cerebelli; and MS: Medulla spinalis. Scale bars, $50 \mu \mathrm{m}$.

\subsection{Vascular Histology of the Brain}

To determine whether BPA affects blood vessels and induces atherosclerosis in the brain, brain histological analysis was conducted. In the diencephalon area, the blood vessels in the control group had large vascular lumens and were filled with red blood cells, but the blood vessels in the BPA exposure group showed vascular occlusion and had only few red blood cells (Figure 7C,D); the images of the H\&E staining of atherosclerotic pathology was referred to in Paralichthys albigutta (Figure S3). In the midbrain, there were large fissures between blood vessels and the surrounding tissue in the treatment group (Figure $7 \mathrm{~F}, \mathrm{~N}$ ), but no fissures were observed in the control group (Figure 7E,N). Fissures were also observed around blood vessels in the telencephalon region and around capillaries in the diencephalon and corpus cerebellum regions after BPA exposure (Figure $7 \mathrm{H}, \mathrm{J}, \mathrm{L}$ ); statistical analysis showed that the number of fissures blood vessels per unit area was also higher in the BPA treatment group (Figure $7 \mathrm{~N}-\mathrm{Q}$ ), but no fissures were observed in these areas in the control group brains (Figure 7G,I,K). Additionally, we examined the vessel wall thickness in similar anatomical areas and found that the blood vessel walls were thicker in the BPA-treated group than in the control 
group (Table 1). These results suggested that BPA exposure might damage brain blood vessels and induce atherosclerosis.
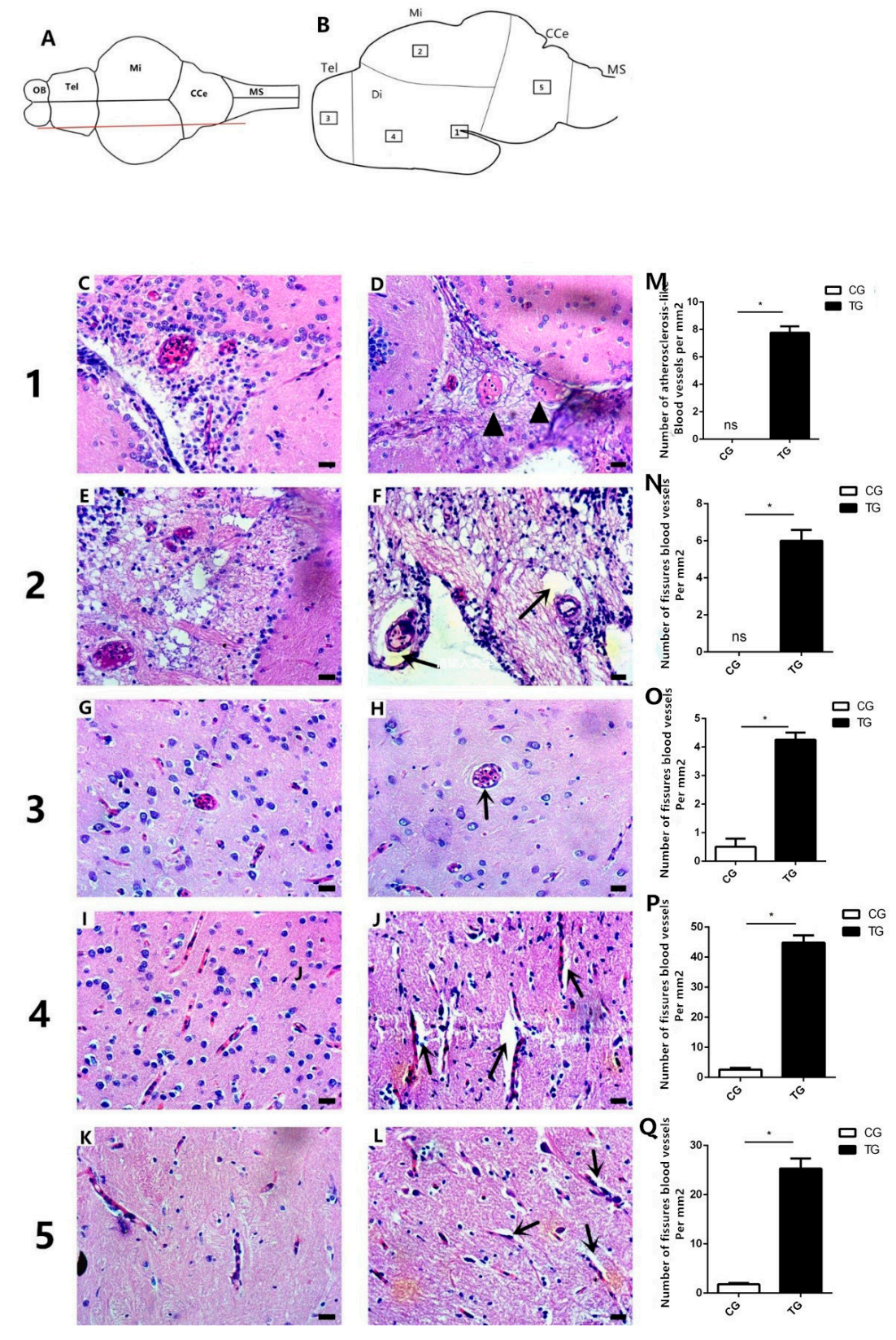

Figure 7. Histology of the brains of goldfish from the control group $\left(0 \mu \mathrm{g} \mathrm{L}^{-1}\right)$ and the BPA treatment group $\left(50 \mu \mathrm{g} \mathrm{L}^{-1}\right)$. (A) Dorsal view of the fish brain; the red line represents the slice position. (B) Longitudinal sectional views of the fish brain; the boxed areas represent the positions of the sections. (C-L) Histology of the brain in the five areas. $(\mathrm{C}, \mathrm{E}, \mathrm{G}, \mathrm{I}, \mathrm{K})$ Brain histology of the five areas in the control group. ( $\mathbf{D}, \mathbf{F}, \mathbf{G}, \mathbf{J}, \mathbf{L})$ Brain histology of the five areas in the BPA-exposed group. Atherosclerosis was found in the diencephalon (black arrowheads) in the BPA-exposed group (D). Fissures (black arrows) were found in the midbrain (F) and telencephalon (G) region, as well as in capillaries in the telencephalon (J) and corpus cerebelli (L). (M) Statistical analysis of the number of fissured blood vessels per unit area. ${ }^{*}$ indicates a significant difference $(n=3) .(\mathbf{N}-\mathbf{Q})$ Statistical analysis of the number of atherosclerosis-like vessels per $\mathrm{mm}^{2}$. Five images per fish were analyzed. * indicates a significant difference $(n=3)$. Tel: Telencephalon; OB: Olfactory bulb; Di: Diencephalon; Mi: Midbrain; CCe: Corpus cerebelli; and MS: Medulla spinalis. Scale bars, $50 \mu \mathrm{m}$. 
Table 1. Summary of vessel wall thicknesses in similar anatomical areas for the control group (CG) and treatment group (TG).

\begin{tabular}{cccccc}
\hline \multirow{2}{*}{ Sample } & \multicolumn{5}{c}{ Areas } \\
\cline { 2 - 6 } & $\mathbf{1}$ & $\mathbf{2}$ & $\mathbf{3}$ & $\mathbf{4}$ & $\mathbf{5}$ \\
\hline CG & $3.52 \pm 0.07 \mu \mathrm{m}^{*}$ & $3.73 \pm 0.07 \mu \mathrm{m} *$ & $0.89 \pm 0.03 \mu \mathrm{m}$ & $0.81 \pm 0.03 \mu \mathrm{m}$ & $0.92 \pm 0.02 \mu \mathrm{m}$ \\
\hline TG & $2.31 \pm 0.04 \mu \mathrm{m}$ & $2.54 \pm 0.05 \mu \mathrm{m}$ & $0.71 \pm 0.02 \mu \mathrm{m}$ & $0.62 \pm 0.01 \mu \mathrm{m}$ & $0.67 \pm 0.02 \mu \mathrm{m}$ \\
\hline
\end{tabular}

The results are expressed as the mean $\pm \mathrm{SD}(n=3)$. Significant differences between CG and TG are identified with * Numbers 1, 2, 3, 4, and 5 correspond to the brain anatomical areas shown in Figure 7.

\section{Discussion}

BPA is widely found in aquatic environments and causes reproductive toxicity in fish, often resulting in abnormal testicular and ovarian development [16-18,28,29]. BPA has also been demonstrated to accumulate in various fish tissues, leading to pathological changes, including changes in the endocannabinoid system, changes in the ontogeny of the cortisol stress response, and energetic impairments $[22,23,30]$.

Several reports have shown the neurotoxic effects of BPA in a variety of animal models [20,31-37]. However, the toxic effects of BPA on fish brain development and related gene expression have rarely been studied. In the present study, we found that BPA disrupts dopaminergic processes, including increasing the apoptosis of dopamine neurons enriched brain regions, decreasing serum dopamine concentrations, and downregulating genes involved in dopaminergic signaling pathways. Studies have indicated that dopamine neurons are vulnerable to oxidative damage, and many studies have shown that BPA is an exogenous estrogen with oxidative toxicity and it can cause tissue oxidative damage [38-44]. Thus, we propose that BPA may affect dopamine synthesis via oxidative damage to dopamine neurons. Furthermore, we detected the increase of apoptosis in dopamine neurons-enriched brain regions by TUNEL, and the results show that BPAs have oxidative damage and may cause the apoptosis of dopamine neurons. In addition, the dopamine system is complex, and its normal function involves many components, such as dopamine metabolic enzymes (TH, MAO), the dopamine transporter (DAT), dopamine receptor (DR), and three signaling pathways of the dopamine system. Changes in any of these factors affect dopamine synthesis. Our results showed that BPA exposure significantly decreased the serum dopamine concentrations and the expressions of th, mao, dat, dr1, and $d r 2$. These changes may explain the effects of BPA that induced the damage (TUNEL) to the dopamine neurons and further induced the decrease of dopamine levels and the expression of genes involved in dopamine synthesis. There is a possibility that some TUNEL-positive cells are not dopaminergic cells, because the loss of D1 receptors is also found in addition to DAT and D2. Thus, further research is needed.

In the present study, the KEGG pathway enrichment analysis was shown to be toxic to blood vessels in the brains of BPA-exposed fish. After BPA exposure, the expression of genes related to stable blood flow with laminar shear stress ( $h s p 90, c a l m, \beta$-catenin, and F-actin) was decreased, while the expressions of mek 5 and $e t-1$, genes related to disturbed blood flow with low/oscillatory shear stress, was increased, suggesting that BPA exposure weakened the stabilization of blood flow and strengthened the disruption of blood flow. Furthermore, several DEGs relating to the immune function were altered, especially in pathways relating to herpes simplex infections. These results suggest that BPA may affect immune functions in fish; however, further research is needed to establish this.

The shape of blood vessels is strongly influenced by blood shear stress, with shape changes preferentially occurring in areas of disturbed flow or low shear stress [45-48]. The shear stress of blood in the brain is affected by vascular ECs (Endothelial Cells); these ECs, which cover the inner surface of blood vessels, are constantly exposed to shear stress because of the frictional force created by blood flow. Previous studies have shown that altered gene expression in ECs changes EC caveolae, inducing changes in local shear stress $[49,50]$. In addition, shear stress can alter smooth muscle cells and affect 
vascular smooth muscle contraction [51,52], thus weakening vascular smooth muscle contraction and causing blood vessel deformation (such as the fissures observed in the brain histological analysis) (Figure 7). In addition, a previous study has shown that increases in fluid shear stress increased mek5 expression in ECs [53]. Our study showed that, after BPA exposure, mek5 expression was increased, indicating that the vascular pressure in goldfish brain was increased. The vascular histology showed that the brain blood vessels had atherosclerosis in BPA-exposed fish. We propose that BPA exposure may disrupt gene expression in vascular ECs and thus weaken vascular smooth muscle contraction, destroying the shape of blood vessels and resulting in atherosclerosis, but more research may be needed.

\section{Materials and Methods}

\subsection{Animals and Treatments}

Juvenile goldfish (mean body length, $6.5 \pm 0.69 \mathrm{~cm}$; mean body weight, $9.4 \pm 1.23 \mathrm{~g}$ ) were obtained from a fish farm in Guangzhou, China, and were maintained in aquaria. The concentration of BPA used in this study was $50 \mu \mathrm{g} \mathrm{L}^{-1}$. The exposure experiments were carried out in six different glass tanks; 3 tanks were used for each exposure group, with 30 fish per tank. The treated group was exposed to $50 \mu \mathrm{g} \mathrm{L}-1$ BPA for 30 days. After the exposure, 15 fish were randomly selected from each group, anesthetized with MS-222 (Sigma-Aldrich, Saint Louis, MO, USA) and then sacrificed via decapitation. The brains of three fish in each group were collected, and the total RNA was extracted from each brain. The RNA from the brains of three fish from each tank was mixed into one pooled RNA sample for a total of three pooled samples per group. Then, the six pooled RNA samples (three control group samples and three treatment group samples) were used to construct a transcriptome library. The brains of six of the fifteen fish sacrificed from each group were dissected and fixed for histological analysis. The brains of the other 6 fish were collected, flash-frozen in liquid nitrogen, and then stored at $-80{ }^{\circ} \mathrm{C}$. At the same time, blood samples were collected, and pairs of samples were mixed to form pooled serum samples after centrifugation. All animal experiments were conducted in accordance with the guidelines and approval was obtained from the appropriate Animal Research and Ethics Committees of South China Agricultural University (SHAUQ201810, 15 April 2018).

\subsection{RNA Extraction and Library Preparation}

The brain samples were lysed in TRIzol reagent (Invitrogen, Carlsbad, CA, USA) for RNA extraction according to the manufacturer's instructions. Agarose gels (1.5\%) were used to examine RNA degradation and contamination. RNA concentrations and integrity were examined with a Qubit RNA Assay Kit in a Qubit 2.0 Fluorometer (Life Technologies, Carlsbad, CA, USA) and with an RNA Nano 6000 Assay Kit in an Agilent Bioanalyzer 2100 System (Agilent Technologies, Santa Clara, CA, USA).

RNA ( $2 \mu \mathrm{g}$ per sample) was used as input material for RNA sample preparation. In short, mRNA was isolated from total RNA using magnetic beads with bound poly(T) oligonucleotides, and the purified mRNA was then cut into small fragments at high temperatures using bivalent cations.

First-strand cDNA was synthesized with a random hexamer primer using the M-MuLV Reverse Transcriptase kit (MBI, Fermentas, Lithuania); Second-strand cDNA was then synthesized for the first-strand fragments. Double-stranded cDNA was synthesized using a SuperScript double-stranded cDNA synthesis kit (Invitrogen, Carlsbad, CA, USA) with random hexamer primers (Illumina). Then, the synthesized cDNA was subjected to end-repair, phosphorylation and ' $A$ ' base addition according to Illumina's library construction protocol. cDNA fragments of 150-200 bp were preferentially selected, and PCR amplification was performed for 15 cycles. Finally, the PCR products were purified and evaluated using an Illumina HiSeq 2500 platform.

\subsection{Transcriptome Assembly and Gene Functional Annotation}

Raw reads were obtained after the removal of reads containing the adaptor sequence, reads containing poly-N sequences and low-quality reads with SQ values $<20$ and nucleotide ratios 
greater than 50\%. Only sequences with Phred quality scores $>20$ were selected for downstream analysis. The selected reads were assembled into transcripts using Trinity software [54]. Then, TGICL V2.1 software [55] was used to process the contigs; redundant sequences were removed and additional assembly was performed.

Unigenes were 'blasted' (searched) against the Nr database, the Kyoto Encyclopedia of Genes and Genomes (KEGG) database, the Swiss-Prot database, and the EuKaryotic Orthologous Groups (KOG)/Clusters of Orthologous Groups (COG) database using BLASTx. In addition, the protein sequences were obtained, and the functional annotation information was obtained according to the protein sequences.

\subsection{Differential Expression Analysis}

Differential expression analysis was identified using DESeq2 version 1.4.5 [56]. The Benjamini and Hochberg method was used to adjust the obtained $p$-values [57]. Genes with fold changes $>2$ and $p<0.05$ were considered differentially expressed.

\subsection{Gene Ontology (GO) and KEGG Enrichment Analyses}

GOseq V1.16.2 software was used to perform GO enrichment analysis of differentially expressed genes (DEGs) [58]. Then, KOBAS software was used to test the statistical enrichment of the DEGs in the KEGG pathways [59].

\subsection{RNA Isolation, Reverse Transcription and $q R T-P C R$}

Total RNA was extracted using TRIzol reagent and then reverse transcribed using a Transcriptor First Strand cDNA Synthesis Kit (Toyobo, Tsuruga, Japan) according to the manufacturer's instructions. SYBR Green I Master Mix (Roche, Basel, Switzerland) was used to conduct qRT-PCR analysis on a Roche LightCycler 480 Real-Time PCR System. The PCR conditions were as follows: $95^{\circ} \mathrm{C}$ for $5 \mathrm{~min}$ for activation followed by 40 cycles of $95^{\circ} \mathrm{C}$ for $20 \mathrm{~s}, 58^{\circ} \mathrm{C}$ for $20 \mathrm{~s}$, and $72{ }^{\circ} \mathrm{C}$ for $20 \mathrm{~s}$. The ef1 gene was used as the internal control. The expression of each target gene was normalized by the $2^{-\Delta \Delta \mathrm{Ct}}$ method [60]. The primers are listed in Table S6.

\subsection{Dopamine Detection}

Serum dopamine was measured using a Fish Dopamine ELISA Kit (Cusabio, Wuhan, China), following the manufacturer's instructions. A Synergy H4 Hybrid Multi-Mode Microplate Reader (BioTek, Winusky, USA) was used to detect the optical density per well at $450 \mathrm{~nm}$ within $10 \mathrm{~min}$.

\subsection{Brain Histology}

Brains were dissected from fish in the control group and treatment group, fixed in Bouin's fluid for $24 \mathrm{~h}$ at room temperature, dehydrated, and embedded in paraffin wax. Subsequently, the samples were subjected to histological analysis through hematoxylin and eosin (H \& E) staining.

\subsection{TUNEL Staining}

TUNEL analysis was performed, as described in a previous study [61]. Brain apoptosis was detected with a TUNEL assay kit (Roche, Basel, Switzerland) according to the manufacturer's instructions. After TUNEL staining, hematoxylin was used to restain the tissues. Photographs were obtained under a Nikon optical microscope (Nikon, Sendai, Japan).

\section{Conclusions}

In this study, transcriptome analysis of the brains of goldfish exposed to BPA was performed using RNA-Seq. Upon comparison of unigene expression profiles, 327 unigenes were found to be upregulated and 153 unigenes were found to be downregulated in the BPA exposure group compared 
to the control group. Among these DEGs, dopaminergic signaling pathway-related genes were significantly downregulated in the BPA-exposed group compared to the control group. Furthermore, TUNEL analysis revealed that BPA exposure induced dopamine neuron enriched regions in brain apoptosis, and dopamine concentrations also decreased after BPA exposure, indicating that BPA can disrupt dopaminergic processes. KEGG analysis showed that the DEGs were highly enriched in the fluid shear stress and atherosclerosis pathway. In addition, qRT-PCR analysis of DEGs in the fluid shear stress and atherosclerosis pathway and analysis of the vascular histology of the brain showed that BPA exposure can damage brain blood vessels. We could greatly speculate that BPA exposure globally affects the DA system, particularly in the diencephalon, which is known as the most DA enriched region, and leads to the reduction of serum DA levels in goldfish.

Supplementary Materials: Supplementary materials can be found at http://www.mdpi.com/1422-0067/20/24/6206/s1.

Author Contributions: Q.W., H.Z. and F.L. conceived and designed this study; F.L., Q.H., X.L. and S.X. conducted the experiments; Q.W., L.Z. and H.Y. analyzed the data; Q.W., H.Y. and H.Z. prepared the manuscript.

Funding: This work is supported by the Youth Fund Project of the National Natural Science Foundation of China (No. 41806151), the National Natural Science Foundation of China (No. 31972768), the Guangdong Provincial Natural Science Foundation (2017A030313099), and the Provincial Projects with Special Funds for Promoting Economic Development of Marine and Fisheries Department of Guangdong (SDYY-2018-05).

Acknowledgments: We would also like to acknowledge the Support of Young Science and Technology Talent Training Fund of South China Agricultural University.

Conflicts of Interest: The authors declare no conflict of interest.

\section{References}

1. Krishnan, A.V.; Stathis, P.; Permuth, S.F.; Tokes, L.; Feldman, D. Bisphenol-A: An estrogenic substance is released from polycarbonate flasks during autoclaving. Endocrinology 1993, 132, 2279-2286. [CrossRef]

2. Vandenberg, L.N.; Maffini, M.V.; Sonnenschein, C.; Rubin, B.S.; Soto, A.M. Bisphenol-A and the great divide: a review of controversies in the field of endocrine disruption. Endocr. Rev. 2009, 30, 75-95. [CrossRef] [PubMed]

3. Grand View Research, Inc. Biosurfactants Market by Product (Market Research Report Code: GVR20). Available online: http://www.grandviewresearch.com.sci-hub.org/industry-analysis/biosurfactants-industry (accessed on 1 April 2014).

4. Belfroid, A.; van Velzen, M.; van der Horst, B.; Vethaak, D. Occurrence of bisphenol a in surface water and uptake in fish: evaluation of field measurements. Chemosphere 2002, 49, 97-103. [CrossRef]

5. Yamamoto, T.; Yasuhara, A.; Shiraishi, H.; Nakasugi, O. Bisphenol a in hazardous waste landfill leachates. Chemosphere 2001, 42, 415-418. [CrossRef]

6. Howdeshell, K.L.; Peterman, P.H.; Judy, B.M.; Taylor, J.A.; Orazio, C.E.; Ruhlen, R.L.; vom Saal, F.S.; Welshons, W.V. Bisphenol a is released from used polycarbonate animal cages into water at room temperature. Environ. Health Perspect. 2003, 111, 1180-1187. [CrossRef]

7. Huang, Y.Q.; Wong, C.K.; Zheng, J.S.; Bouwman, H.; Barra, R.; Wahlstrom, B.; Neretin, L.; Wong, M.H. Bisphenol A (BPA) in China: a review of sources, environmental levels, and potential human health impacts. Environ. Int. 2012, 42, 91-99.

8. Zhang, Y.Y.; Gao, J.C.; Xu, P.; Yuan, C.; Qin, F.; Liu, S.Z.; Zheng, Y.; Yang, Y.P.; Wang, Z.Z. Low-dose bisphenol a disrupts gonad development and steroidogenic genes expression in adult female rare minnow gobiocypris rarus. Chemosphere 2014, 112, 435-442. [CrossRef]

9. Carlisle, J.; Chan, D.; Golub, M.; Henkel, S.; Painter, P.; Lily, W.K. Toxicological Profile for Bisphenol A; Office of Environmental Health Hazard Assessment: Sacramento, CA, USA, 2009.

10. Gavrilescu, M.; Demnerova, K.; Aamand, J.; Agathos, S.; Fava, F. Emerging pollutants in the environment: present and future challenges in biomonitoring, ecological risks and bioremediation. New Biotechnol. 2015, 32, 147-156. [CrossRef]

11. Michalowicz, J. Bisphenol A-sources, toxicity and biotransformation. Environ. Toxicol. Pharmacol. 2014, 37, 738-758. [CrossRef] 
12. Syranidou, E.; Christofilopoulos, S.; Politi, M.; Weyens, N.; Venieri, D.; Vangronsveld, J.; Kalogerakis, N. Bisphenol-A removal by the halophyte Juncus acutus in a phytoremediation pilot: characterization and potential role of the endophytic community. J. Hazard. Mater. 2017, 323, 350-358. [CrossRef]

13. Zhang, W.; Yin, K.; Chen, L. Bacteria-mediated bisphenol A degradation. Appl. Microbiol. Biotechnol. 2013, 97, 5681-5689. [CrossRef]

14. Kang, J.H.; Katayama, Y.; Kondo, F. Biodegradation or metabolism of bisphenol A: from microorganisms to mammals. Toxicology 2006, 217, 81-90. [CrossRef] [PubMed]

15. Hodson, P.V. Indicators of ecosystem health at the species level and the example of selenium effects on fish. Environ. Monit. Assess. 1990, 15, 241-254. [CrossRef] [PubMed]

16. Wang, Q.; Yang, H.R.; Yang, M.; Yu, Y.P.; Yan, M.T.; Zhou, L.; Liu, X.C.; Xiao, S.Q.; Yang, Y.; Wang, Y.X.; et al. Toxic effects of bisphenol A on goldfish gonad development and the possible pathway of BPA disturbance in female and male fish reproduction. Chemosphere 2019, 221, 235-245. [CrossRef] [PubMed]

17. Prisco, M.; Liguoro, A.; Comitato, R.; Cardone, A.; D'Onghia, B.; Ricchiari, L.; Angelini, F.; Andreuccetti, P. Apoptosis during spermatogenesis in the spotted ray Torpedo marmorata. Mol. Reprod. Dev. 2003, 64,341-348. [CrossRef]

18. Rhee, J.S.; Kim, B.M.; Lee, C.J.; Yoon, Y.D.; Lee, Y.M.; Lee, J.S. Bisphenol A modulates expression of sex differentiation genes in the self-fertilizing fish, Kryptolebias marmoratus. Aquat. Toxicol. 2011, 104, 218-229. [CrossRef]

19. Huang, Q.S.; Chen, Y.J.; Lin, L.F.; Liu, Y.Y.; Chi, Y.L.; Lin, Y.; Ye, G.Z.; Zhu, H.M.; Dong, S.J. Different effects of bisphenol a and its halogenated derivatives on the reproduction and development of Oryzias melastigma under environmentally relevant doses. Sci. Total. Environ. 2017, 595, 752-758. [CrossRef]

20. Molina, A.; Abril, N.; Moralesprieto, N.; Monterde, J.; Ayala, N.; Lora, A.; Moyano, R. Hypothalamic-pituitary-ovarian axis perturbation in the basis of bisphenol a (bpa) reproductive toxicity in female zebrafish (danio rerio). Ecotox. Environ. Safe. 2018, 156, 116-124. [CrossRef]

21. Li, D.; Chen, Q.; Cao, J.L.; Chen, H.X.; Xia, L.L.; Cedergree, N.; Xie, H.B.; Xie, L.T. The chronic effects of lignin-derived bisphenol and bisphenol A in Japanese medaka Oryzias latipes. Aquat. Toxicol. 2016, 170, 199-207. [CrossRef]

22. Birceanu, O.; Mai, T.; Vijayan, M.M. Maternal transfer of bisphenol A impacts the ontogeny of cortisol stress response in rainbow trout. Aquat. Toxicol. 2015, 168, 11-18. [CrossRef]

23. Nagato, E.G.; Simpson, A.J.; Simpson, M.J. Metabolomics reveals energetic impairments in Daphnia magna exposed to diazinon, malathion and bisphenol-A. Aquat. Toxicol. 2016, 170, 175-186. [CrossRef]

24. Wang, X.; Dong, Q.; Chen, Y.; Jiang, H.; Xiao, Q.; Wang, Y.; Li, W.; Bai, C.; Huang, C.; Yang, D. Bisphenol A affects axonal growth, musculature and motor behavior in developing zebrafish. Aquat. Toxicol. 2013, 142-143, 104-113. [CrossRef] [PubMed]

25. Jones, D.C.; Miller, G.W. The effects of environmental neurotoxicants on the dopaminergic system: a possible role in drug addiction. Biochem. Pharmacol. 2008, 76, 569-581. [CrossRef] [PubMed]

26. Matsui, H. Dopamine system, cerebellum, and nucleus ruber in fish and mammals. Develop. Growth Differ. 2017, 59, 219-227. [CrossRef] [PubMed]

27. Schweitzer, J.; Lö hr, H.; Alida Filippi, A.; Driever, W. Dopaminergic and Noradrenergic Circuit Development in Zebrafish. Dev. Neurobiol. 2012, 72, 256-268. [CrossRef] [PubMed]

28. Chen, J.; Xiao, Y.; Gai, Z.; Li, R.; Zhu, Z.; Bai, C.; Tanguay, R.L.; Xu, X.; Huang, C.; Dong, Q. Reproductive toxicity of low level bisphenol A exposures in a two-generation zebrafish assay: Evidence of male-specific effects. Aquat. Toxicol. 2015, 169, 204-214. [CrossRef]

29. Tamschick, S.; Rozenblut-Kościsty, B.; Ogielska, M.; Lehmann, A.; Hoffmann, P.F.; Lutz, I.; Schneider, R.J.; Kloas, W.; Stöck, M. Impaired gonadal and somatic development corroborate vulnerability differences to the synthetic estrogen ethinylestradiol among deeply diverged anuran lineages. Aquat. Toxicol. 2016, 177, 503-514. [CrossRef]

30. Forner-Piquer, I.; Santangeli, S.; Maradonna, F.; Verde, R.; Piscitelli, F.; di Marzo, V.; Habibi, H.R.; Carnevali, O. Role of Bisphenol A on the Endocannabinoid System at central and peripheral levels: Effects on adult female zebrafish. Chemosphere 2018, 205, 118-125. [CrossRef]

31. Honma, T.; Miyagawa, M.; Suda, M.; Wang, R.S.; Kobayashi, K.; Sekiguchi, S. Effects of perinatal exposure to bisphenol A on brain neurotransmitters in female rat offspring. Ind. Health. 2006, 44, 510-524. [CrossRef] 
32. Matsuda, S.; Matsuzawa, D.; Ishii, D.; Tomizawa, H.; Sutoh, C.; Nakazawa, K.; Amano, K.; Sajiki, J.; Shimizu, E. Effects of perinatal exposure to low dose of bisphenol A on anxiety like behavior and dopamine metabolites in brain. Prog. Neuropsychopharmacol. Biol. Psychiat. 2012, 39, 273-279.

33. Elsworth, J.D.; Jentsch, J.D.; Vandevoort, C.A.; Roth, R.H.; Redmond, D.E.; Leranth, C., Jr. Prenatal exposure to bisphenol A impacts midbrain dopamine neurons and hippocampal spine synapses in non-human primates. Neurotoxicology 2013, 35, 113-120. [CrossRef]

34. Castro, B.; Sanchez, P.; Miranda, M.T.; Torres, J.M.; Ortega, E. Identification of dopamine- and serotonin-related genes modulated by bisphenol A in the prefrontal cortex of male rats. Chemosphere 2015, 139, 235-239. [CrossRef] [PubMed]

35. Chen, F.; Zhou, L.; Bai, Y.; Zhou, R.; Chen, L. Hypothalamic-pituitary-adrenal axis hyperactivity accounts for anxiety- and depression-like behaviors in rats perinatally exposed to bisphenol A. J. Biomed. Res. 2015, 29, 250-258. [PubMed]

36. Canonicolau, J.; Vaillant, C.; Pellegrini, E.; Charlier, T.D.; Kah, O.P. Coumailleau Estrogenic Effects of Several BPA Analogs in the Developing Zebrafish Brain. Front. Neurosci. 2016, 10, 112.

37. Drobná, Z.; Henriksen, A.D.; Wolstenholme, J.T.; Montiel, C.; Rissman, E.F.; Lambeth, P.S.; Shang, S.; Harris, E.P.; Zhou, C.; Flaws, J.A.; et al. Transgenerational effects of bisphenol a on gene expression and dna methylation of imprinted genes in brain. Endocrinology 2017, 159, 132-144. [CrossRef] [PubMed]

38. Wang, X.K.; Michaelis, E.K. Selective neuronal vulnerability to oxidative stress in the brain. Front. Aging Neurosci. 2010, 30, 12. [CrossRef]

39. Mehwish, F.; Parvez, L.K. Oxidative stress and histopathologic biomarkers of exposure to bisphenol-a in the freshwater fish, ctenopharyngodon idella. Braz. J. Pharm. Sci. 2018, 53, e17003.

40. Gassman, N.R. Induction of oxidative stress by bisphenol A and its pleiotropic effects. Environ. Mol. Mutagen. 2017, 58, 60-71. [CrossRef]

41. Liu, Z.W.; Zhou, T.; Ziegler, A.C.; Dimitrion, P.; Zuo, L. Oxidative Stress in Neurodegenerative Diseases: From Molecular Mechanisms to Clinical Applications. Oxid. Med. Cell. Longev. 2017, 2017, 2525967. [CrossRef]

42. Hassan, Z.K.; Elobeid, M.A.; Virk, P.; Omer, S.A.; ElAmin, M.; Daghestani, M.H.; AlOlayan, E.M. Bisphenol A induces hepatotoxicity through oxidative stress in rat model. Oxid. Med. Cell. Longev. 2012, 2012, 194829. [CrossRef]

43. Lv, Y.; Lu, S.; Dai, Y.; Rui, C.; Wang, Y.; Zhou, Y.; Li, Y.; Pang, Q.; Fan, R. Higher dermal exposure of cashiers to BPA and its association with DNA oxidative damage. Environ. Int. 2017, 98, 69-74. [CrossRef]

44. Li, S.; Jin, Y.; Zhao, H.; Jiang, Y.; Cai, Z. Evaluation of bisphenol A exposure induced oxidative RNA damage by liquid chromatography-mass spectrometry. Chemosphere 2019, 222, 235-242. [CrossRef] [PubMed]

45. Traub, O.; Berk, B.C. Laminar shear stress: mechanisms by which endothelial cells transduce an atheroprotective force. Arterioscler. Thromb. Vasc. Biol. 1988, 18, 677-685. [CrossRef] [PubMed]

46. Gimbrone, M.A.; Topper, J.N., Jr.; Nagel, T.; Anderson, K.R.; Garcia-Cardena, G. Endothelial dysfunction, hemodynamic forces, and atherogenesis. Ann. N. Y. Acad. Sci. 2000, 902, 230-239. [CrossRef] [PubMed]

47. Cheng, C.; Tempel, D.; van Haperen, R.; van der Baan, A.; Grosveld, F.; Daemen, M.J.; Krams, R.; de Crom, R. Atherosclerotic lesion size and vulnerability are determined by patterns of fluid shear stress. Circulation 2006, 113, 2744-2753. [CrossRef] [PubMed]

48. Nigro, P.; Abe, J.; Berk, B.C. Flow shear stress and atherosclerosis: a matter of site specificity. Antioxid. Redox. Signal. 2011, 15, 1405-1414. [CrossRef] [PubMed]

49. Chien, S. Mechanotransduction and endothelial cell homeostasis: the wisdom of the cell. Am. J. Physiol. Heart. Circ. Physiol. 2007, 292, 1209-1224. [CrossRef]

50. Davies, P.F.; Civelek, M.; Fang, Y.; Fleming, I. The atherosusceptible endothelium: endothelial phenotypes in complex haemodynamic shear stress regions in vivo. Cardiovasc. Res. 2013, 99, 315-327. [CrossRef]

51. Nam, D.; Ni, C.W.; Rezvan, A.; Suo, J.; Budzyn, K.; Llanos, A.; Harrison, D.; Giddens, D.; Jo, H. Partial carotid ligation is a model of acutely induced disturbed flow, leading to rapid endothelial dysfunction and atherosclerosis. Am. J. Physiol. Heart. Circ. Physiol. 2009, 297, 1535-1543. [CrossRef]

52. Zhou, J.; Li, Y.S.; Chien, S. Shear stress-initiated signaling and its regulation of endothelial function. Arterioscler. Thromb. Vasc. Biol. 2014, 34, 2191-2198. [CrossRef]

53. Li, L.; Tatake, R.J.; Natarajan, K.; Taba, Y.; Garin, G.; Tai, C.; Leung, E.; Surapisitchat, J.; Yoshizumi, M.; Yan, C.; et al. Fluid shear stress inhibits TNF-mediated JNK activation via MEK5-BMK1 in endothelial cells. Biochem. Biophys. Res. Commun. 2008, 370, 159-163. [CrossRef] 
54. Grabherr, M.G.; Haas, B.J.; Yassour, M.; Levin, J.Z.; Thompson, D.A.; Amit, I.; Adiconis, X.; Fan, L.; Raychowdhury, R.; Zeng, Q.; et al. Full-length transcriptome assembly from RNA-Seq data without a reference genome. Nat. Biotechnol. 2011, 29, 644-652. [CrossRef] [PubMed]

55. Pertea, G.; Huang, X.; Liang, F.; Antonescu, V.; Sultana, R.; Karamycheva, S.; Lee, Y.; White, J.; Cheung, F.; Parvizi, B.; et al. TIGR Gene Indices clustering tools (TGICL): a software system for fast clustering of large EST datasets. Bioinformatics 2003, 19, 651-652. [CrossRef] [PubMed]

56. Love, M.I.; Huber, W.; Anders, S. Moderated estimation of fold change and dispersion for RNA-seq data with DESeq2. Genome. Biol. 2014, 15, 550. [CrossRef] [PubMed]

57. Benjamini, Y.; Hochberg, Y. Controlling the false discovery rate: a practical and powerful approach to multiple testing. J. R. Stat. Soc. 1995, 57, 289-300. [CrossRef]

58. Young, M.D.; Wakefield, M.J.; Smyth, G.K.; Alicia, O. Gene ontology analysis for RNA-seq: Accounting for selection bias. Genome. Biol. 2010, 11, R14. [CrossRef]

59. Mao, X.; Cai, T.; Olyarchuk, J.G.; Wei, L. Automated genome annotation and pathway identification using the KEGG Orthology (KO) as a controlled vocabulary. Bioinformatics 2005, 21, 3787-3793. [CrossRef]

60. Livak, K.J.; Schmittgen, T.D. Analysis of relative gene expression data using realtime quantitative PCR and the 2(-Delta Delta C(T)) method. Methods 2001, 25, 402-408. [CrossRef]

61. Wang, Q.; Liu, Y.; Peng, C.; Wang, X.; Xiao, L.; Wang, D.D.; Chen, J.X.; Zhang, H.H.; Zhao, H.F.; Li, S.S.; et al. Molecular regulation of sex change induced by methyltestosterone -feeding and methyltestosterone-feeding withdrawal in the protogynous orange-spotted grouper. Biol. Reprod. 2017, 97, 324-333. [CrossRef]

(C) 2019 by the authors. Licensee MDPI, Basel, Switzerland. This article is an open access article distributed under the terms and conditions of the Creative Commons Attribution (CC BY) license (http://creativecommons.org/licenses/by/4.0/). 